\title{
Association between neutrophil-to-lymphocyte ratio and parathyroid hormone in patients with primary hyperparathyroidism
}

\author{
Hung-Bun Lam ${ }^{1}$, Po-Sheng Yang ${ }^{1}$, Ming-Nan Chien², Jie-Jen Lee ${ }^{1,3}$, Li-Fen Chao ${ }^{4}$, Shih-Ping Cheng ${ }^{1,3}$
}

\author{
${ }^{1}$ Department of Surgery, MacKay Memorial Hospital and Mackay Medical College, \\ Taipei, Taiwan \\ ${ }^{2}$ Division of Endocrinology and Metabolism, Department of Internal Medicine, MacKay \\ Memorial Hospital and Mackay Medical College, Taipei, Taiwan \\ ${ }^{3}$ Graduate Institute of Medical Sciences and Department of Pharmacology, \\ Taipei Medical University, Taipei, Taiwan \\ ${ }^{4}$ Department of Nursing, Chronic Diseases and Health Promotion Research Center, \\ Chang Gung University of Science and Technology, Taoyuan, Taiwan
}

Submitted: 23 December 2017

Accepted: 11 March 2018

Arch Med Sci 2019; 15 (4): 880-886

DOI: https://doi.org/10.5114/aoms.2018.74758

Copyright (c) 2018 Termedia \& Banach

\section{Abstract}

Introduction: Primary hyperparathyroidism (PHPT) is associated with adverse cardiovascular outcomes which may result from an increase in systemic inflammation. Previously we have shown that serum parathyroid hormone (PTH) levels are independently associated with inflammatory indicators. The neutrophil-to-lymphocyte ratio (NLR) is an inexpensive, widely available marker of inflammation. In the present study, we aimed to assess the longitudinal changes in NLR before and after parathyroidectomy.

Material and methods: This retrospective study included 95 patients diagnosed with PHPT who underwent parathyroidectomy between 2006 and 2016. Follow-up complete blood counts were available in 31 patients.

Results: At diagnosis, 43 (45\%) patients presented with overt clinical symptoms and had higher serum calcium and PTH levels. Preoperative NLR was positively correlated with total white blood cell count $(p=0.001)$, serum calcium $(p=0.001)$, and PTH level $(p=0.013)$. The NLR was not associated with sex, age, comorbidities, or parathyroid weight. Among patients who were cured of PHPT, the median NLR decreased from 2.26 to 1.77 after parathyroidectomy $(p=0.037)$. There was no difference in hemoglobin, total white blood cells, or platelet count before and after surgery.

Conclusions: We found a positive correlation of preoperative NLR with calcium and PTH levels in PHPT patients. After curative parathyroidectomy, NLR modestly decreased without changes in other hematological parameters.

Key words: inflammation, neutrophil-to-lymphocyte ratio, parathyroid hormone, primary hyperparathyroidism, parathyroidectomy.

\section{Introduction}

Parathyroid hormone (PTH) plays an important role in the control of calcium homeostasis. Accumulating evidence suggests a complex interplay between inflammation and PTH regulation. Enhanced PTH secretion has been observed in septic patients [1]. Furthermore, in the general population, serum PTH levels are independently associated with several

\author{
Corresponding author: \\ Shih-Ping Cheng MD, PhD \\ Department of Surgery \\ MacKay Memorial \\ Hospital 92, Section 2 \\ Chung-Shan North Road \\ Taipei 10449, Taiwan \\ Phone: +8862 25433535 \\ Fax: +88622723 3897 \\ E-mail: surg.mmh@gmail.com
}


inflammatory markers [2]. It is well known that inflammation participates in the pathogenesis of cardiovascular events. Large community-based studies reported that PTH level was associated with the degree of atherosclerosis and the risk of clinically overt atherosclerotic disease [3]. Nonetheless, it remains controversial whether PTH is an independent risk marker for cardiovascular disease $[4,5]$.

Primary hyperparathyroidism (PHPT) is the most common cause of outpatient hypercalcemia due to excessive PTH secretion from the enlarged parathyroid gland(s). Cardiac remodeling is common in patients with PHPT and is associated with lowgrade inflammation [6]. Even mild PHPT may be associated with subclinical carotid vascular manifestations [7]. However, the reported effects of parathyroidectomy on inflammation are not consistent across studies. Using S100A4 and the soluble receptor SCD14 as surrogate markers, a Norwegian study of 57 PHPT patients demonstrated that parathyroidectomy may partially reverse the systemic inflammation [8]. In contrast, a Swedish study reported that interleukin-6 (IL-6), C-reactive protein (CRP), and erythrocyte sedimentation rate increased 1 year after surgery in parallel with a decrease in cardiac function [9]. Although the discrepancies may reflect heterogeneity among study populations, additional studies are warranted to determine the influence of surgical treatment of PHPT on systemic inflammatory status.

The neutrophil-to-lymphocyte ratio (NLR) is a readily available and inexpensive biomarker of systemic inflammation $[10,11]$. It has been shown that although total white blood cell count is an independent predictor of cardiovascular death or myocardial infarction, NLR has greater predictability [12]. Subsequent studies confirmed that NLR is an independent predictor of short- and longterm mortality in patients with acute coronary syndromes [13]. In addition, a high NLR is associated with inferior survival in many solid tumors [14]. The aim of this study was to investigate the association of NLR with clinical and biochemical variables for testing the hypothesis that PHPT is related to the state of systemic inflammation. Furthermore, we evaluated the effects of parathyroidectomy on the longitudinal changes in NLR.

\section{Material and methods}

This study was a retrospective review of a prospectively maintained database on all parathyroid procedures performed at MacKay Memorial Hospital, Taipei, Taiwan. Institutional review board approval was obtained, with patient consent waived. Inclusion criteria were adult patients who underwent initial parathyroidectomy for sporadic PHPT between 2006 and 2016. Patients with hereditary
PHPT, including multiple endocrine neoplasia type 1 and 2A, familial isolated hyperparathyroidism, and hyperparathyroidism-jaw tumor syndrome, were not included. Exclusion criteria included parathyroid carcinoma $(n=3)$, reoperative parathyroidectomy $(n=4)$, and chronic kidney disease stage 3 or higher $(n=2)$. Finally, 95 patients constituted the study cohort.

The PHPT diagnosis was based on biochemical abnormalities characterized by the persistence of high normal or elevated calcium levels and non-suppressed serum PTH levels. Serum intact PTH (1-84) was measured as previously described [15]. All patients underwent neck ultrasonography and sestamibi imaging with or without single-photon emission computed tomography (SPECT) before surgery. Nearly one-half of patients were operated on for overt clinical symptoms, including recurrent nephrolithiasis and fragility fracture. On rare occasions, patients presented with hypercalcemic crisis and were initially treated with aggressive volume expansion and bisphosphonates. Thereafter, parathyroidectomy was performed on an elective basis. The indications for surgery in otherwise asymptomatic patients were according to the International Workshop guidelines if any one of the following criteria was fulfilled [16]: serum calcium > $1 \mathrm{mg} / \mathrm{dl}$ upper normal limit; $T$-score $<-2.5$ by dual-energy X-ray absorptiometry; estimated glomerular filtration rate or creatinine clearance $<60 \mathrm{ml} / \mathrm{min}$ or reduced by $>30 \%$ from expected; and age $<50$ years.

Data collected included patient demographics, laboratory studies, preoperative imaging studies, surgical approach, and postoperative outcomes. Patients routinely had complete blood counts with automated differential counts on the day before surgery. NLR was calculated as the neutrophil count divided by the lymphocyte count, both obtained from the same blood sample [17]. Serum calcium and PTH levels were obtained on the first postoperative day. After the initial follow-up, serum calcium and PTH levels were measured at 6 and 12 months and then annually. Persistent PHPT was defined as hypercalcemia with inappropriately elevated PTH levels noted within 6 months after parathyroidectomy. Patients were defined as having recurrent PHPT if hypercalcemia developed beyond 6 months after surgery.

Complete blood counts were not included in our routine follow-up examinations. We manually searched the hospital laboratory database for any complete blood counts available after postoperative 6 months. Data which were obtained during the diagnosis and/or treatment for apparently infectious or inflammatory disorders were excluded. Postoperative NLR was calculated in the same manner as preoperatively. 
Table I. Comorbidities of the study cohort

\begin{tabular}{|lc|}
\hline Comorbidity & Patient, $\boldsymbol{n}$ (\%) \\
\hline Hypertension & $31(33)$ \\
\hline Diabetes mellitus & $14(15)$ \\
\hline Malignancy & $5(5)$ \\
\hline Chronic hepatitis & $5(5)$ \\
\hline Hyperthyroidism & $2(2)$ \\
\hline Systemic lupus erythematosus & $1(1)$ \\
\hline
\end{tabular}

\section{Statistical analysis}

All results are expressed as median (interquartile ranges - IQR) for continuous variables and as the number of cases (percentage) for categorical variables. Data were analyzed using the $\chi^{2}$ and Fisher's exact tests when appropriate. Unpaired and paired continuous data were compared using the twotailed Mann-Whitney $U$ and Wilcoxon signed-rank tests, respectively. Spearman's rank correlation analysis was used to identify possible relationships among the variables [18]. Statistical analysis was performed using STATA statistical software package version 14.0 (StataCorp, College Station, TX, USA).

\section{Results}

The study cohort consisted of 95 consecutive PHPT patients who fulfilled the inclusion and exclusion criteria. The median age at diagnosis was 59 years (IQR: $51-67)$, and 72 (76\%) were women. Comorbidities of the study cohort are shown in Table I. About one-third of patients had hyperten- sion. Five patients had prior history of malignancy, including breast cancer $(n=3)$, rectal cancer $(n=1)$, and melanoma $(n=1)$. A total of $43(45 \%)$ patients presented with overt clinical symptoms, including nephrolithiasis $(n=32)$, fracture $(n=5)$, hypercalcemic crisis $(n=3)$, pancreatitis $(n=2)$, and stroke $(n=1)$. In general, symptomatic patients were younger and less likely to be female (Table II).

The serum calcium and PTH levels of the study cohort were $11.4 \mathrm{mg} / \mathrm{dl}$ (IQR: 10.7-12.2) and 148 pg/ml (IQR: 113-264), respectively. As expected, symptomatic patients had higher calcium and PTH levels. Furthermore, PTH levels were positively correlated with serum calcium levels and negatively correlated with hemoglobin and phosphorus levels (Table III). There was no difference in biochemical parameters between genders. At surgery, 83 (87\%) patients were found to have single gland disease, while 12 (13\%) had multigland disease. The median removed parathyroid weight was $586 \mathrm{mg}$ (IQR: 313-1213). The parathyroid weight was closely correlated with preoperative PTH levels (Spearman's rho $=0.589, p<0.0001$ ).

Preoperatively, the median white blood cell count for the entire group of patients was $7000 / \mu \mathrm{l}$ (IQR: 5700-8500). Two patients presented with mild leukocytosis (12 000 and $12800 / \mu$ l) before parathyroidectomy. The NLR at diagnosis was relatively low, with a median of 2.20 (IQR: 1.662.81). There was no difference in NLR between patients with and without comorbidities ( $p=$ 0.642 ), and symptomatic and asymptomatic patients had similar NLR values $(p=0.200)$. Overall,

Table II. Patient characteristics grouped by initial presentation

\begin{tabular}{|c|c|c|c|}
\hline Parameter & Asymptomatic $(n=52)$ & Symptomatic $(n=43)$ & $P$-value \\
\hline Female, $n(\%)$ & $44(85)$ & $28(65)$ & 0.027 \\
\hline Age [years] & $62(56-68)$ & $56(48-62)$ & 0.003 \\
\hline Body mass index $\left[\mathrm{kg} / \mathrm{m}^{2}\right]$ & $23.7(21.3-26.3)$ & $23.7(22.0-27.1)$ & 0.427 \\
\hline Body weight [kg] & $58(52-66)$ & $60(54-66)$ & 0.244 \\
\hline Hemoglobin [g/dl] & $12.8(11.6-13.6)$ & $13.6(11.9-14.7)$ & 0.025 \\
\hline White blood cells $[/ \mu l]$ & $6650(5950-7650)$ & $7500(5500-9000)$ & 0.220 \\
\hline Platelet count $\left[\times 10^{3} / \mu \mathrm{l}\right]$ & $237(174-272)$ & $240(194-296)$ & 0.340 \\
\hline Serum calcium [mg/dl] & $10.9(10.7-11.6)$ & $11.6(11.0-12.5)$ & 0.023 \\
\hline Serum phosphorus [mg/dl] & $3.2(2.8-3.4)$ & $2.8(2.6-3.3)$ & 0.065 \\
\hline Parathyroid hormone [pg/ml] & $131(100-181)$ & $189(116-380)$ & 0.030 \\
\hline Parathyroid weight [mg] & $443(266-980)$ & $614(367-2237)$ & 0.187 \\
\hline Multigland disease, $n(\%)$ & $6(12)$ & $6(14)$ & 0.724 \\
\hline Neutrophil-to-lymphocyte ratio & $2.04(1.59-2.62)$ & $2.27(1.78-2.86)$ & 0.200 \\
\hline
\end{tabular}

Data are given as frequencies (percentages) or medians (interquartile ranges). 
Table III. Spearman rank correlation coefficients between clinical and laboratory parameters in 95 patients with primary hyperparathyroidism

\begin{tabular}{|lcccccccc|}
\hline Parameter & BMI & Hb & WBC & Platelets & Ca & P & PTH & NLR \\
\hline Age & 0.054 & $-0.379^{* * *}$ & -0.060 & -0.209 & 0.019 & 0.181 & 0.037 & -0.023 \\
\hline BMI & & $0.224^{*}$ & $0.234^{*}$ & 0.081 & 0.036 & -0.119 & -0.005 & -0.131 \\
\hline $\mathrm{Hb}$ & & $0.219^{*}$ & $0.252^{*}$ & $-0.232^{*}$ & 0.001 & $-0.369^{\star *}$ & -0.185 \\
\hline WBC & & & $0.364^{\star *}$ & 0.075 & -0.008 & -0.095 & $0.352^{* *}$ \\
\hline Platelets & & & & 0.090 & -0.063 & 0.060 & 0.078 \\
\hline Ca & & & & & $-0.328^{* *}$ & $0.672^{* * *}$ & $0.376^{* *}$ \\
\hline P & & & & & & $-0.337^{* *}$ & -0.123 \\
\hline PTH & & & & & & & $0.274^{*}$ \\
\hline
\end{tabular}

$B M I$ - body mass index, $H b$ - hemoglobin, NLR - neutrophil-to-lymphocyte ratio, PTH - parathyroid hormone, WBC - white blood cells. ${ }^{*}$ Spearman's correlation $p<0.05,{ }^{* *} p<0.01,{ }^{* * *} p<0.001$.

the NLR before parathyroidectomy was positively correlated with total white blood cell count $(p=$ $0.001)$, serum calcium $(p=0.001)$, and PTH level $(p=0.013)$. The associations of NLR with calcium and PTH levels are depicted in Figure 1. There was no correlation between NLR and sex $(p=0.178)$, age $(p=0.834)$, body mass index (BMI, $p=0.237)$, serum phosphorus level ( $p=0.281)$, or parathyroid weight $(p=0.241)$. Of interest, the median NLR of single gland disease and multigland disease was 2.21 and 1.68, respectively. The NLR of multigland disease was marginally lower than that of single gland disease ( $p=0.054)$.

After parathyroidectomy, 4 (4\%) patients were found to have persistent disease. Recurrent disease was identified in 1 patient. Follow-up complete blood counts (at least 6 months after surgery) were available in 31 (33\%) patients. In a patient who had persistent disease but declined reoperation, his NLR increased from 1.49 before surgery to 2.26 at follow-up. For the remaining disease-free patients $(n=30)$, the median NLR at diagnosis and at follow-up was 2.26
(IQR: 1.70-3.00) and 1.77 (IQR: 1.59-2.61), respectively. As shown in Figure 2, postoperative NLR was significantly lower than preoperative NLR in patients who were cured of PHPT ( $p=0.037)$. There was no difference in hemoglobin $(p=0.570)$, total white blood cells $(p=0.131)$, or platelet count $(p=0.505)$ before and after surgery.

\section{Discussion}

A number of observational studies have noted a relationship between PTH levels and the presence of an inflammatory state [19]. In patients with PHPT, endothelium-dependent vasodilation could be impaired despite normal intima-media thickness [20]. It is therefore suggested that endothelial dysfunction may contribute to increased cardiovascular morbidity and mortality in PHPT. Recently, Stamatelopoulos et al. reported that PHPT was an independent determinant of peripheral and aortic diastolic blood pressure [21]. However, the association with systolic blood pressure was lost after adjusting for CRP. This implies that
A

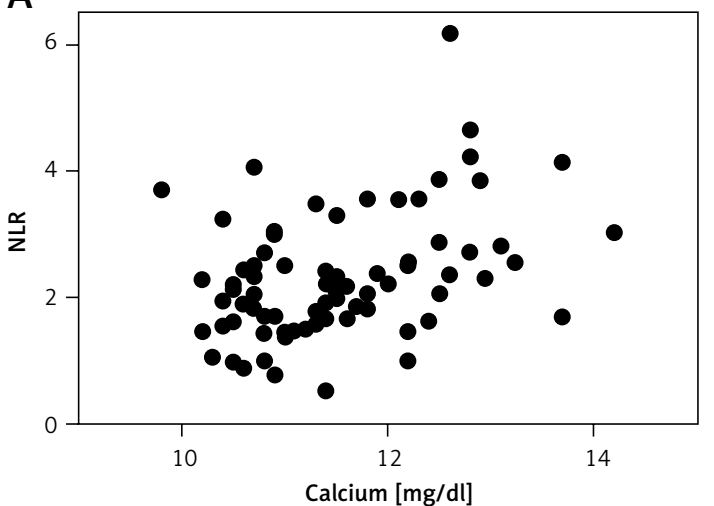

B

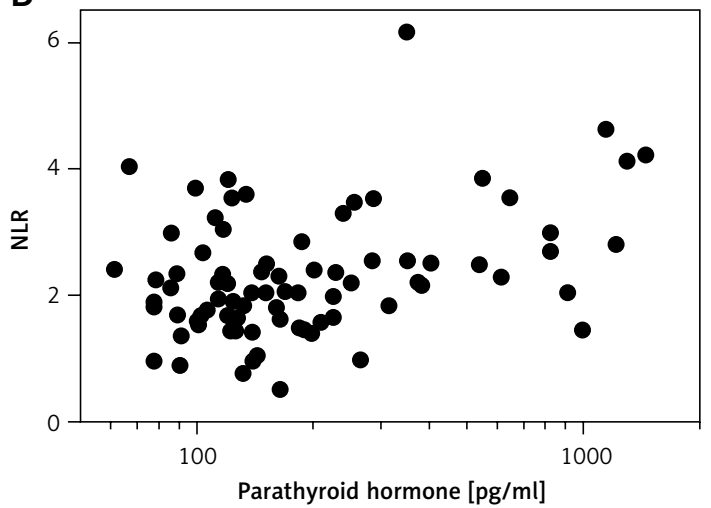

Figure 1. Scatter plots of neutrophil-to-lymphocyte ratio (NLR) versus serum calcium (A; Spearman's rho $=0.376$, $p=0.001)$ and parathyroid hormone level (B; Spearman's rho $=0.274, p=0.013$ ) in 95 patients with primary hyperparathyroidism 


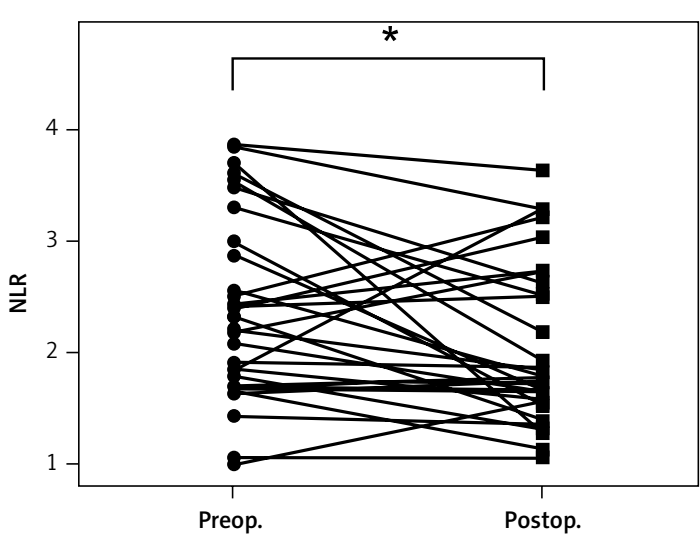

Figure 2. Neutrophil-to-lymphocyte ratio (NLR) before and after curative parathyroidectomy in 30 patients with primary hyperparathyroidism

*Wilcoxon signed-rank test $p<0.05$.

PHPT may increase blood pressure through PTH and inflammatory-mediated mechanisms.

In the present study, we found that NLR was positively correlated with serum calcium and PTH levels. Our findings are partially consistent with those of Zeren et al. [22]. The authors noted that NLR had a better correlation with the parathyroid adenoma size than with PTH levels. However, we did not observe a correlation between NLR and the parathyroid weight. The discrepancy may result from the fact that the authors included patients with parathyroid cancer (3 out of 32, i.e. about $9 \%$ ), while we excluded such patients from the study. Furthermore, given the small volume of most parathyroid tumors, we believed that weight is a more accurate measure than the tumor diameter. In addition, the authors employed the Pearson correlation test for the analysis of variables with apparently skewed distribution.

The benefits of surgery for patients with mild asymptomatic PHPT are still a matter of debate. Compared to active surveillance, we have shown that parathyroidectomy may be associated with better quality of life, especially in the emotional aspects of well-being [23]. Nonetheless, definitive evidence is still lacking to support the effectiveness of parathyroidectomy in altering cardiovascular outcomes in patients with asymptomatic PHPT [24]. Many studies have shown that successful parathyroidectomy leads to an improvement in endothelial, vascular, and cardiac dysfunction in symptomatic and asymptomatic subjects $[25,26]$. A recent meta-analysis further revealed that parathyroidectomy may reduce left ventricular mass [27]. By contrast, some investigators reported no significant differences in blood pressure, inflammation parameters, and cardiovascular surrogate markers among mild PHPT patients randomized to either observation or surgery [28]. These conflicting results may be due to differences in the duration and severity of PHPT, and the length of follow-up. It is reasonable to hypothesize that mild PHPT may be associated with early and reversible alterations, while prolonged dysfunctions caused by more severe PHPT are resistant to surgical intervention. In this regard, we noted a small, but significant, decrease in NLR following parathyroidectomy even though a substantial portion of our patients were clinically symptomatic at presentation. This is in keeping with the results obtained for parathyroidectomy performed for severe secondary hyperparathyroidism [29]. Future research is needed to examine whether our findings can be translated into more clinically relevant outcomes.

The mechanisms underlying PHPT-associated inflammation are not clear. The shoulder region of atherosclerotic plaques was characterized by strong immunostaining of PTH-related protein (PTHrP), the type 1 PTH/PTHrP receptor, and monocyte chemoattractant protein-1 [30]. Such expression was colocalized with inflammatory cells. Furthermore, the expression of the receptor for advanced glycation end products (RAGE) as well as IL- 6 was increased in umbilical vein endothelial cells following stimulation with clinically relevant concentrations of PTH [31]. These observations suggest that PTH may have direct proinflammatory effects on the vasculature. Moreover, PTH regulates the hematopoietic niche by inducing hematopoietic cell expansion and migration [32]. Other indirect mechanisms may also be important in PHPT-associated inflammation. For example, circulating fibroblast growth factor 23 (FGF23) levels are increased in PHPT and decreased after parathyroidectomy [33]. Recent studies indicate that FGF23 possesses proinflammatory and immune-modulatory properties [34]. Adipose tissue is another possible factor mediating the association between PHPT and inflammation. Subcutaneous fat tissue from the neck of PHPT patients expressed increased levels of genes involved in immunity and inflammatory processes [35]. Several lines of evidence suggest that BMI and/or visceral adiposity are positively correlated with PTH concentrations [36, 37]. Nonetheless, we observed a weak correlation between BMI and total white blood cell count but not NLR in this study.

Another interesting finding in the present study was a negative correlation between hemoglobin and PTH levels. This is in keeping with the experience of others [38]. Anemia is common in patients with symptomatic PHPT and may be associated with marrow fibrosis [39]. Although reversal of anemia and marrow fibrosis has been shown after parathyroidectomy, we found that hemoglobin levels did not significantly differ before and after surgery. Additionally, PHPT-associated thrombocytopenia has been reported in the literature [40]. In this regard, we did not detect an association between PTH and platelet count in the present 
study or any changes in the platelet count following parathyroidectomy.

Consistent with previous reports [7, 8], women were more likely to have PHPT than men in this study. Although men had a higher incidence of clinical symptoms, both sexes had similar PTH levels and NLR at presentation. Limitations of our study include the small sample size and its retrospective nature. It should be borne in mind that this study is a single-center study conducted in a medical center in Taiwan, where calcium screening is not included in routine biochemical tests. Thus, the percentage of asymptomatic PHPT patients would be lower than that of other countries. In addition, our study was limited by its relatively short follow-up period. The number of cases with persistent or recurrent PHPT was too small for statistical evaluation. Finally, vitamin D levels were not routinely determined in our patients. Vitamin D deficiency is a potential confounding factor for some of the associations found in this study.

In conclusion, our study demonstrated a positive correlation of NLR with preoperative calcium and PTH levels in PHPT patients, suggesting the presence of an association between PHPT and systemic inflammation. After curative parathyroidectomy, NLR modestly decreased, without changes in other hematological parameters. Our findings support a cause-and-effect relationship between PHPT and inflammation, and systemic inflammation may be reversible after prompt therapy.

\section{Acknowledgments}

This study was supported by a research grant (MOST-106-2314-B-195-010-MY3) from the Ministry of Science and Technology of Taiwan.

\section{Conflict of interest}

The authors declare no conflict of interest.

\section{References}

1. Lind L, Carlstedt F, Rastad J, et al. Hypocalcemia and parathyroid hormone secretion in critically ill patients. Crit Care Med 2000; 28: 93-9.

2. Cheng SP, Liu CL, Liu TP, Hsu YC, Lee JJ. Association between parathyroid hormone levels and inflammatory markers among US adults. Mediators Inflamm 2014; 2014: 709024.

3. Hagstrom E, Michaelsson K, Melhus H, et al. Plasmaparathyroid hormone is associated with subclinical and clinical atherosclerotic disease in 2 community-based cohorts. Arterioscler Thromb Vasc Biol 2014; 34: 1567-73.

4. Folsom AR, Alonso A, Misialek JR, et al. Parathyroid hormone concentration and risk of cardiovascular diseases: the Atherosclerosis Risk in Communities (ARIC) study. Am Heart J 2014; 168: 296-302.

5. Wannamethee SG, Welsh P, Papacosta O, Lennon L, Whincup PH, Sattar N. Elevated parathyroid hormone, but not vitamin $\mathrm{D}$ deficiency, is associated with in- creased risk of heart failure in older men with and without cardiovascular disease. Circ Heart Fail 2014; 7: 732-9.

6. Verheyen N, Meinitzer A, Grubler MR, et al. Low-grade inflammation and tryptophan-kynurenine pathway activation are associated with adverse cardiac remodeling in primary hyperparathyroidism: the EPATH trial. Clin Chem Lab Med 2017; 55: 1034-42.

7. Walker MD, Fleischer J, Rundek T, et al. Carotid vascular abnormalities in primary hyperparathyroidism. J Clin Endocrinol Metab 2009; 94: 3849-56.

8. Christensen MH, Fenne IS, Nordbo Y, et al. Novel inflammatory biomarkers in primary hyperparathyroidism. Eur J Endocrinol 2015; 173: 9-17.

9. Almqvist EG, Bondeson AG, Bondeson L, Svensson J. Increased markers of inflammation and endothelial dysfunction in patients with mild primary hyperparathyroidism. Scand J Clin Lab Invest 2011; 71: 139-44.

10. Ozcicek A, Ozcicek F, Yildiz G, et al. Neutrophil-to-lymphocyte ratio as a possible indicator of epicardial adipose tissue in patients undergoing hemodialysis. Arch Med Sci 2017; 13: 118-23.

11. Yin Y, Kuai S, Liu J, et al. Pretreatment neutrophil-to-lymphocyte ratio in peripheral blood was associated with pulmonary tuberculosis retreatment. Arch Med Sci 2017; 13: 404-11.

12. Horne BD, Anderson JL, John JM, et al.; Intermountain Heart Collaborative Study Group. Which white blood cell subtypes predict increased cardiovascular risk? J Am Coll Cardiol 2005; 45: 1638-43.

13. Tamhane UU, Aneja S, Montgomery D, Rogers EK, Eagle KA, Gurm HS. Association between admission neutrophil to lymphocyte ratio and outcomes in patients with acute coronary syndrome. Am J Cardiol 2008; 102: 653-7.

14. Templeton AJ, MCNamara MG, Seruga B, et al. Prognostic role of neutrophil-to-lymphocyte ratio in solid tumors: a systematic review and meta-analysis. J Natl Cancer Inst 2014; 106: dju124.

15. Cheng SP, Yang TL, Lee JJ, et al. Gender differences among patients with secondary hyperparathyroidism undergoing parathyroidectomy. J Surg Res 2011; 168: 82-7.

16. Bilezikian JP, Brandi ML, Eastell R, et al. Guidelines for the management of asymptomatic primary hyperparathyroidism: summary statement from the Fourth International Workshop. J Clin Endocrinol Metab 2014; 99: 3561-9.

17. Liu CL, Lee JJ, Liu TP, Chang YC, Hsu YC, Cheng SP. Blood neutrophil-to-lymphocyte ratio correlates with tumor size in patients with differentiated thyroid cancer. J Surg Oncol 2013; 107: 493-7.

18. Huang SS, Liao YC, Wang WF. The factor structure for the geriatric depression scale in screening depression in Taiwanese patients with very mild to moderate dementia. Int J Gerontol 2017; 11: 36-40.

19. Alemzadeh R, Kichler J. Parathyroid hormone is associated with biomarkers of insulin resistance and inflammation, independent of vitamin D status, in obese adolescents. Metab Syndr Relat Disord 2012; 10: 422-9.

20. Kosch M, Hausberg M, Vormbrock K, Kisters K, Rahn KH, Barenbrock M. Studies on flow-mediated vasodilation and intima-media thickness of the brachial artery in patients with primary hyperparathyroidism. Am J Hypertens 2000; 13: 759-64.

21. Stamatelopoulos K, Athanasouli F, Pappa T, et al. Hemodynamic markers and subclinical atherosclerosis in 
postmenopausal women with primary hyperparathyroidism. J Clin Endocrinol Metab 2014; 99: 2704-11.

22. Zeren S, Yaylak F, Ozbay I, Bayhan Z. Relationship between the neutrophil to lymphocyte ratio and parathyroid adenoma size in patients with primary hyperparathyroidism. Int Surg 2015; 100: 1185-9.

23. Cheng SP, Lee JJ, Liu TP, et al. Quality of life after surgery or surveillance for asymptomatic primary hyperparathyroidism: a meta-analysis of randomized controlled trials. Medicine (Baltimore) 2015; 94: e931.

24. Macfarlane DP, Yu N, Leese GP. Subclinical and asymptomatic parathyroid disease: implications of emerging data. Lancet Diabetes Endocrinol 2013; 1: 329-40.

25. Agarwal G, Nanda G, Kapoor A, et al. Cardiovascular dysfunction in symptomatic primary hyperparathyroidism and its reversal after curative parathyroidectomy: results of a prospective case control study. Surgery 2013; 154: 1394-403.

26. Cansu GB, Yilmaz N, Ozdem S, et al. Parathyroidectomy in asymptomatic primary hyperparathyroidism reduces carotid intima-media thickness and arterial stiffness. Clin Endocrinol (Oxf) 2016; 84: 39-47.

27. McMahon DJ, Carrelli A, Palmeri N, et al. Effect of parathyroidectomy upon left ventricular mass in primary hyperparathyroidism: a meta-analysis. J Clin Endocrinol Metab 2015; 100: 4399-407.

28. Bollerslev J, Rosen T, Mollerup CL, et al.; SIPH Study Group. Effect of surgery on cardiovascular risk factors in mild primary hyperparathyroidism. J Clin Endocrinol Metab 2009; 94: 2255-61.

29. Yang PS, Liu CL, Liu TP, Chen HH, Wu CJ, Cheng SP. Parathyroidectomy decreases neutrophil-to-lymphocyte and platelet-to-lymphocyte ratios. J Surg Res 2018; 224: 169-75.

30. Martin-Ventura JL, Ortego M, Esbrit P, HernandezPresa MA, Ortega L, Egido J. Possible role of parathyroid hormone-related protein as a proinflammatory cytokine in atherosclerosis. Stroke 2003; 34: 1783-9.

31. Rashid G, Bernheim J, Green J, Benchetrit S. Parathyroid hormone stimulates endothelial expression of atherosclerotic parameters through protein kinase pathways. Am J Physiol Renal Physiol 2007; 292: F1215-8.

32. Yao H, Miura Y, Yoshioka S, et al. Parathyroid hormone enhances hematopoietic expansion via upregulation of cadherin-11 in bone marrow mesenchymal stromal cells. Stem Cells 2014; 32: 2245-55.

33. Nilsson IL, Norenstedt S, Granath F, Zedenius J, Pernow Y, Larsson TE. FGF23, metabolic risk factors, and blood pressure in patients with primary hyperparathyroidism undergoing parathyroid adenomectomy. Surgery 2016; 159: 211-7.

34. David V, Francis C, Babitt JL. Ironing out the cross talk between FGF23 and inflammation. Am J Physiol Renal Physiol 2017; 312: F1-8.

35. Christensen MH, Dankel SN, Nordbo Y, et al. Primary hyperparathyroidism influences the expression of inflammatory and metabolic genes in adipose tissue. PLoS One 2011; 6: e20481.

36. Cheng SP, Doherty GM, Chang YC, Liu CL. Leptin: the link between overweight and primary hyperparathyroidism? Med Hypotheses 2011; 76: 94-6.

37. George JA, Norris SA, Toman M, Snyman T, Crowther NJ. Visceral adiposity is a predictor of parathyroid hormone levels in healthy adults. J Endocrinol Invest 2016; 39: 447-53.

38. Boxer M, Ellman L, Geller R, Wang CA. Anemia in primary hyperparathyroidism. Arch Intern Med 1977; 137: 588-93.
39. Bhadada SK, Bhansali A, Ahluwalia J, Chanukya GV, Behera A, Dutta P. Anaemia and marrow fibrosis in patients with primary hyperparathyroidism before and after curative parathyroidectomy. Clin Endocrinol (Oxf) 2009; 70: 527-32.

40. Bhadada SK, Sridhar S, Ahluwalia J, et al. Anemia and thrombocytopenia improves after curative parathyroidectomy in a patient of primary hyperparathyroidism (PHPT). J Clin Endocrinol Metab 2012; 97: 1420-2. 